\title{
Faunal Monitoring of Ahmedabad Airport as a Part of Bird-Aircraft Strike Hazard (BASH) Study
}

\author{
K. Mokaria ${ }^{1}$, K. Tatu ${ }^{2}$ and R. D. Kamboj ${ }^{3}$ \\ ${ }^{1,2,3}$ Gujarat Ecological Education and Research (GEER) Foundation, Gandhinagar, Gujarat, India \\ *Corresponding Author: kala303@gmail.com, Tel:+91-8980588545
}

Available online at: www.isroset.org

Received: 17/Sept/2018, Accepted: 29/Sept/2018, Online: 31/Oct/2018

\begin{abstract}
Ahmedabad's Sardar Vallabhbhai Patel International (SVPI) airport is known to have a high potential of bird-aircraft collision hazards. For determining the causes and magnitude of the problem and to find out likely solutions, birds, other small animals (mammals and reptiles) and insects were monitored by Gujarat Ecological Education and Research (GEER) Foundation, Gandhinagar during pre-monsoon and monsoon seasons between 2015 and 2017. For monitoring birds, two linear transects were laid, one each along each side of the runway. Besides transect-based bird surveys, bird species were also randomly recorded in other parts of the airport premises and even outside it (within $1 \mathrm{~km}$ belt parallel to the airport boundary). A total of 59 species of birds were encountered within the airport premises that belonged to 30 different families. Of these, 48 species were recorded along the transects. The majority (i.e., 83\%) of the total 59 species were either obligate or facultative insectivorous birds. As the majority of birds were found to be obligatorily or facultatively insectivorous, insect monitoring was also carried out. For recording insects(species/genera and their numbers), Visual Encounter Survey (VES) was carried out in the operational area. A total of 80 species of insects were encountered which belonged to 42 families and 11 orders. Some measures that were suggested by GEER Foundation for reducing the chances of bird-aircraft collision included regulating the growth of grasses through regular grass-cutting preferably before sunrise or after sunset, controlling population of rodents and termites, considering to facilitate artificial grasses/tuft cover. By roping in GEER Foundation for faunal monitoring seasonally SVPI airport authorities have provided an example of ecological concerns in airport management. Other airports in the country should follow this example.
\end{abstract}

Keywords- Birds, Bird Aircraft Strike Hazard (BASH), Black kite, Insects, Sardar Vallabhbhai Patel (SVP) International Airport

\section{INTRODUCTION}

When a bird or a group of birds is struck by an aircraft, it is referred to as a bird strike [1,2]. It is also referred to as BirdAircraft Strike Hazard (BASH). The problem has started when human being invented and started using aircraft, especially the jet aircraft which, due to very high speed, do not give birds an opportunity to escape. BASH poses safety and economic hazards especially during take-off and landing of an aircraft.

From 1999 to 2008, total 71 aircraft accidents occurred due to birdstrikes and 6 of them led to fatal injuries [3]. Considering the value of human life and the huge cost involved in aircraft damage, every effort must be made to minimize the possibilities of BASH. Bird-strike problem should be solved not only to minimize the possibility of loss of human life and loss of money but also to continue the peaceful co-existence by man and birds alike.

Some of the most hazardous birds listed for aircraft flights, which include geese, gulls, pigeons and doves, starlings, sparrows, crows, eagles, hawks, kestrels, and owls $[4,5]$. The reasons for the existence of the birds in the airport area have illustrated in past studies [5]. According to them and some other researchers, birds get attracted to airports for food (e.g. earthworms, termites, grasshoppers, small reptiles/mammals, and seeds), water and shelter. They are also attracted to the airports as they may provide suitable nesting or roosting habitats. Solid waste disposal sites (especially open dump sites) around airports also attract birds though sanitary landfills are less attractive to birds than open dumps [6].

Ahmedabad city's Sardar Vallabhbhai Patel (SVP) International airport in Gujarat State is considered as one of the top-most civilian airports from the viewpoint of the potential of bird-aircraft strike or bird-hit hazard. The rise in the incidences of birdhits at the SVP International airport has communicated the Directorate General of Civil Aviation (DGCA) to declare it a 'critical airport' [7]. To find out the solution to this issue, it was decided by the Airport Environment Management Committee (AEMC) to assign the task of conducting surveys of birds and other fauna (especially entomofauna as many insects constitute 
food of insectivorous birds) to GEER Foundation (an AEMC member). In turn, GEER Foundation carried out surveys during pre-monsoon and monsoon period between 2015 and 2017. It resulted into the comprehensive profile of birds, insects and other fauna occurring at SVP International airport and some insight into causes and likely solution to the threat of BASH at this international airport.

\section{STUDY AREA}

Sardar Vallabhbhai (SVP) Patel International Airport is located in Ahmedabad city of Gujarat State, India(Fig.1). Ahmedabad is the sixth largest city and eighth largest metropolitan area of India, with a metropolitan population of 6.5 million (World Gazetteer. retrieved 11 April 2012). It is the largest city of Gujarat state.

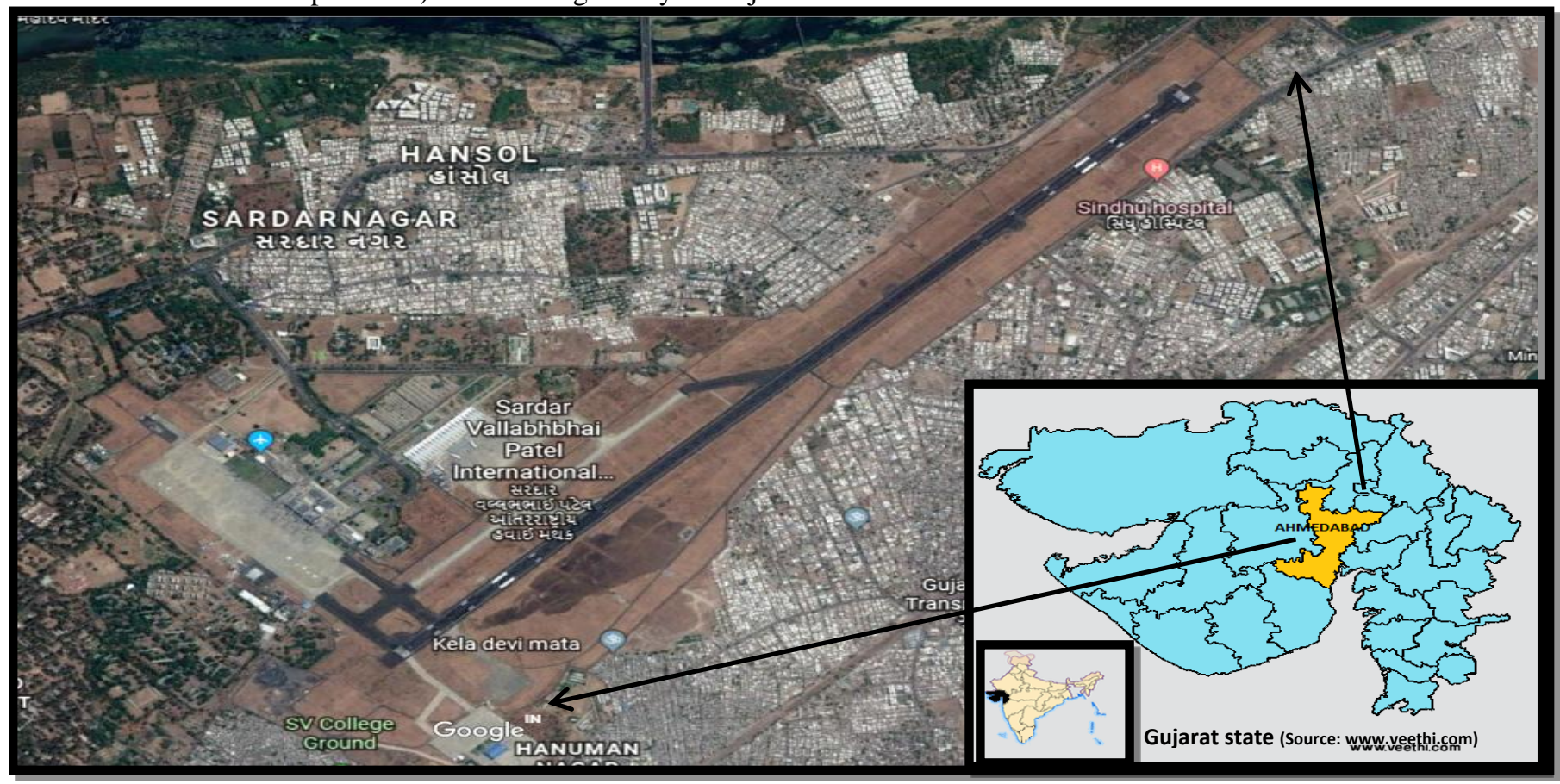

Fig.1 Location of Sardar Vallabhbhai Patel (SVP) international airport, Ahmedabad, Gujarat, India

The Ahmedabad Airport or Sardar Vallabhbhai Patel (SVP) International airport is named after Statesman and former Deputy Prime Minister Shri Sardar Vallabhbhai Patel. The airport is located in the north-east part of Ahmedabad city at $23^{\circ} 04^{\prime} 16.28^{\prime \prime}$ $\mathrm{N}$ Latitude and $072^{\circ} 37^{\prime} 35.15^{\prime \prime}$ E Longitude. It is India's eighth busiest airport with an average of 250 aircraft movements a day. It serves the metropolitan areas of two cities in the State of Gujarat - Ahmedabad, and Gandhinagar. The airport currently consists of four terminals i.e domestic, international, an additional terminal for secondary traffic and a cargo terminal. The airport is spread over an area of $4.55 \mathrm{~km}^{2}$ with the runway measuring $3,600 \mathrm{~m}$. It is situated at the height of $57.61 \mathrm{~m}$ from the Mean Sea Level.

\section{MATERIAL AND METHODS}

Surveys for birds and other fauna (mainly insects and secondarily small mammals and reptiles) were carried out in premonsoon (mainly, early June) and monsoon (mainly, August) seasons between 2015 and 2017. The reason for conducting surveys in pre-monsoon and monsoon was that chances of bird-aircraft hazards would typically increase during this time-frame as per the past trend. For bird (species and population) monitoring, one transect was permanently laid (with its end-points marked with a GPS receiver) on each of the two sides of the runway (Fig.2). 


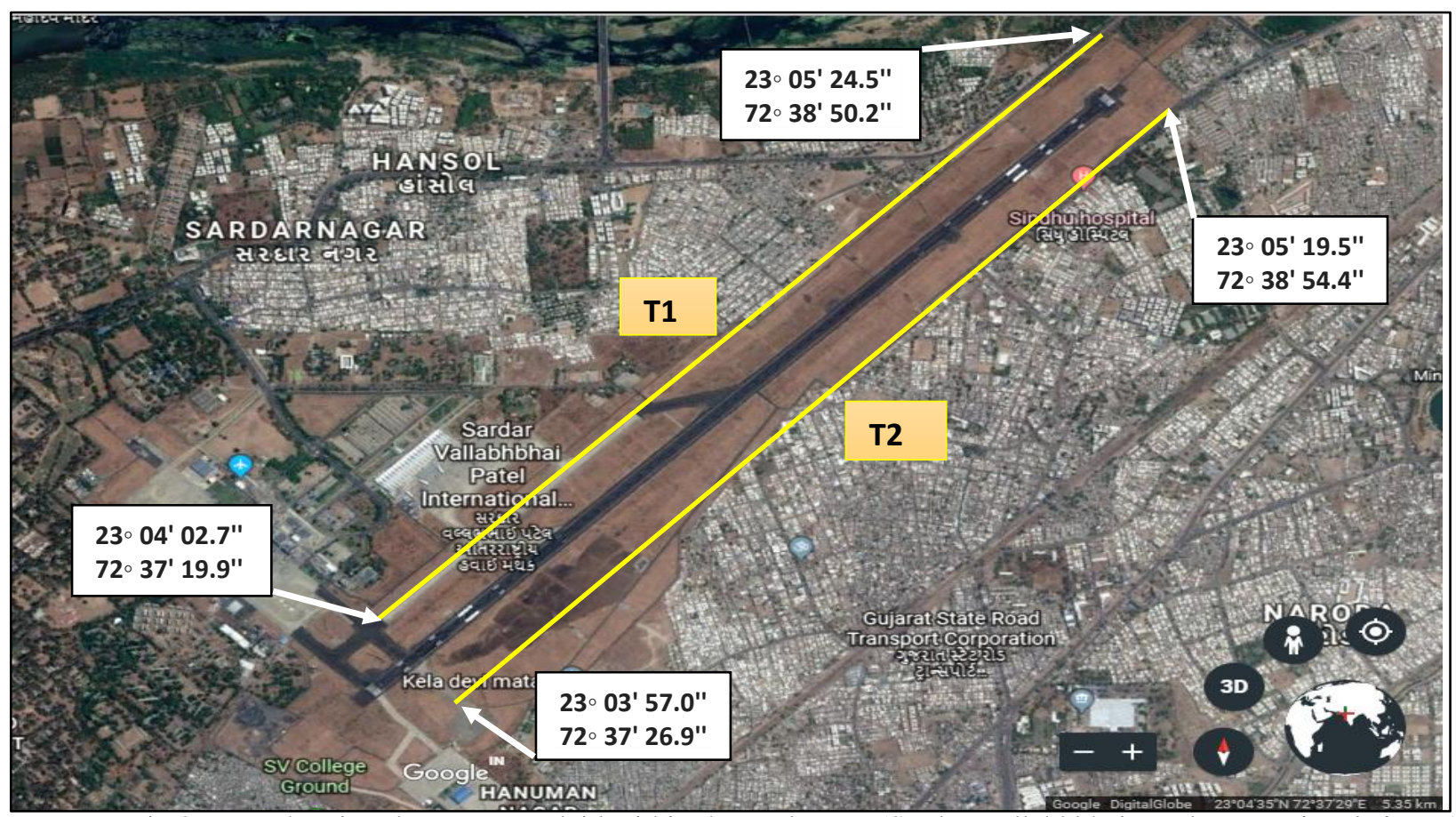

Fig.2: Map showing the Transects laid within the study area (Sardar Vallabhbhai Patel International airport)

Besides species and population monitoring of birds along the two sides of the runway, other parts of the airport premises(at some distance away from the runway) were also assessed for bird species inventorying with the permission and logistic help (especially, a vehicle) of SVPI airport authorities for this purpose, the airport authorities had provided a vehicle. The bird observations were made with the help of a pair of 10X50 binoculars. During each visit to the airport the surveys were conducted from 8 am to $6 \mathrm{pm}$ within the study area and total 80 man-hours were spent for this work each year between 2015 and 2017. The Visual Encounter Survey (VES) approach was adopted for inventorying insects. Total 8 quadrats each of $1 \mathrm{mx} 1 \mathrm{~m}$ laid for insect inventory at equal distance $(500 \mathrm{~m})$ along the transects that were walked for bird inventory. Almost all insects encountered were photographed. Insects were identified using recognized insect identification mannuals/field guides [8, 9,10 and 11].

\section{RESULTS AND DISCUSSION}

A total of 59 species of birds were recorded (Table 1) along the two transects parallel to the runway and at randomly selected observation points within the airport premises. They belonged to 30 different families. It was found that the family Accipitridae(i.e., family of raptors) was represented by a maximum number of species (i.e., 6 species; Fig.3). The family Cisticollidae(i.e., prinias/warblers) was also represented by a relatively good number of species (i.e., 5 species). However, species richness was relatively lower for the rest of the families (Fig.3) 


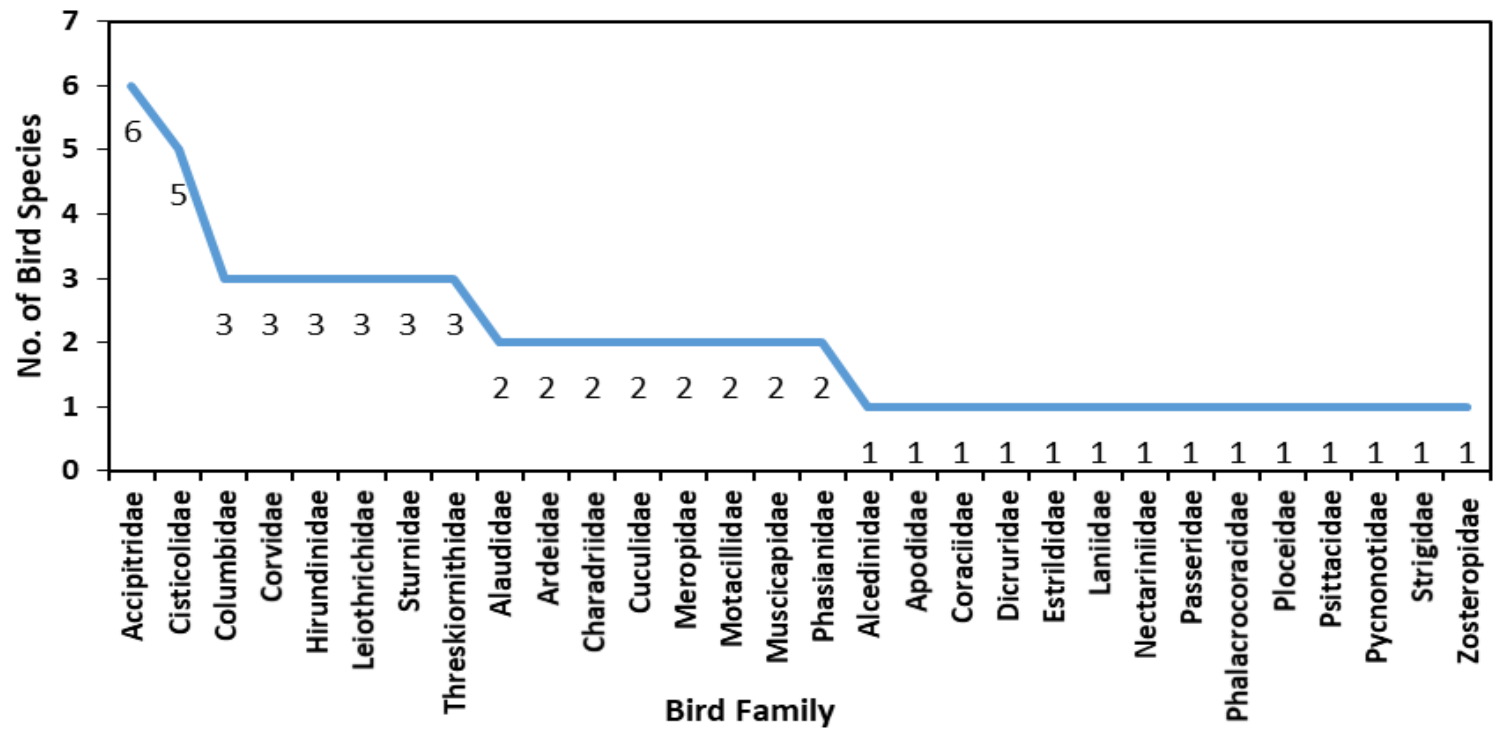

Fig. 3. Familywise species richness of birds recorded at SVP international airport, Ahmedabad

Table 1. Checklist of birds recorded on SVP international airport during pre-monsoon and monsoon period between 2015 and 2017 along with their food items*

\begin{tabular}{|c|l|l|l|l|}
\hline $\begin{array}{c}\text { Sr. } \\
\text { No. }\end{array}$ & \multicolumn{1}{|c|}{ Family } & \multicolumn{1}{|c|}{ Common Name } & \multicolumn{1}{c|}{ Scientific Name } & \multicolumn{1}{c|}{ Food } \\
\hline 1 & Accipitridae & Black Kite & Milvus migrans & $\begin{array}{l}\text { Insects, rodents, reptiles, } \\
\text { Offals }\end{array}$ \\
\hline 2 & Accipitridae & Black-winged Kite & Elanus caeruleus & $\begin{array}{l}\text { Insects, reptiles, rodents, } \\
\text { small birds }\end{array}$ \\
\hline 3 & Accipitridae & Shikra & Accipiter badius & $\begin{array}{l}\text { Rodents, reptiles, birds, } \\
\text { insects }\end{array}$ \\
\hline 4 & Accipitridae & Buzzard sp. & Buteo sp. & Insect a part of the diet \\
\hline 5 & Accipitridae & White-rumped Vulture & Gyps bengalensis & Scavenger \\
\hline 6 & Accipitridae & Booted Hawk Eagle & -- & No insects \\
\hline 7 & Alaudidae & Ashy-crowned Finch- Lark & Eremopterix griseus & Insects(and seeds) \\
\hline 8 & Alaudidae & Rufous-tailed Lark & Ammomanes phoenicura & $\begin{array}{l}\text { insect not main, but a part } \\
\text { of the diet }\end{array}$ \\
\hline 9 & Alcedinidae & White-throated Kingfisher & Halcyon smyrnensis & $\begin{array}{l}\text { Various small animals and } \\
\text { insects }\end{array}$ \\
\hline 10 & Apodidae & House Swift & Apus affinis & Insects \\
\hline 11 & Ardeidae & Cattle Egret & Bubulcus ibis & $\begin{array}{l}\text { Insects, spiders, frogs, } \\
\text { earthworms }\end{array}$ \\
\hline 12 & Ardeidae & Indian Pond Heron & Ardeola grayii & $\begin{array}{l}\text { Fish, amphibians, and } \\
\text { Insects }\end{array}$ \\
\hline 13 & Charadriidae & Red-Wattled Lapwing & Vanellus indicus & Mainly insects \\
\hline 14 & Charadriidae & Yellow-wattled Lapwing & Vanellus malabaricus & Mainly insects \\
\hline 15 & Cisticolidae & Ashy prinia & Prinia socialis & insects \\
\hline 16 & Cisticolidae & Common tailorbird & Orthotomus sutorius & Insects \\
\hline 17 & Cisticolidae & Grey-Breasted Prinia & Prinia hodgsonii & Insects \\
\hline & & & & \\
\hline
\end{tabular}




\begin{tabular}{|c|c|c|c|c|}
\hline 18 & Cisticolidae & Rufous-fronted Prinia & Prinia buchanani & Insects \\
\hline 19 & Cisticolidae & Zitting Cisticola & Cisticola juncidis & Insects \\
\hline 20 & Columbidae & Blue Rock Pigeon & Columba livia & Seeds, Grains \\
\hline 21 & Columbidae & Eurasian Collared Dove & Streptopelia decaocto & Grains, Seeds \\
\hline 22 & Columbidae & Laughing Dove & Streptopelia senegalensis & Grains, seeds \\
\hline 23 & Coraciidae & Indian Roller & Coracias benghalensis & Insects, small vertebrates \\
\hline 24 & Corvidae & House Crow & Corvus splendens & $\begin{array}{l}\text { Various food including } \\
\text { insects }\end{array}$ \\
\hline 25 & Corvidae & Rufous Treepie & Dendrocitta vagabunda & $\begin{array}{l}\text { Rodents, reptiles, small } \\
\text { birds, eggs, Insects }\end{array}$ \\
\hline 26 & Corvidae & Large-billed Crow & Corvus macrorhynchos & $\begin{array}{l}\text { Insects-not main food, but } \\
\text { included in the diet }\end{array}$ \\
\hline 27 & Cuculidae & Asian Koel & Eudynamys scolopaceus & $\begin{array}{l}\text { Insects, (also grain, fruits, } \\
\text { and Others) }\end{array}$ \\
\hline 28 & Cuculidae & Greater Coucal & Centropus sinensis & $\begin{array}{l}\text { Insects, rodents, reptiles, } \\
\text { eggs }\end{array}$ \\
\hline 29 & Dicruridae & Black Drongo & Dicrurus macrocercus & Insects, nector \\
\hline 30 & Estrildidae & Indian Silverbill & Lonchura malabarica & Seeds, grains \\
\hline 31 & Hirundinidae & Dusky Crag Martin & Hirundo concolor & Insects \\
\hline 32 & Hirundinidae & Wire-tailed Swallow & Hirundo smithis & Insectivorous \\
\hline 33 & Hirundinidae & Sand/Plain Martin & Riperia sp. & Insects \\
\hline 34 & Laniidae & Long-tailed Shrike & Lanius scach & Insects \\
\hline 35 & Leiothrichidae & Common Babbler & Turdoides caudatus & Mainly Insects \\
\hline 36 & Leiothrichidae & Large Grey Babbler & Turdoides malcolmi & Mostly Insects \\
\hline 37 & Leiothrichidae & Jungle Babbler & Turdoides striata & Mainly insects \\
\hline 38 & Meropidae & Blue-tailed Bee-eater & Merops phillipensis & Insects \\
\hline 39 & Meropidae & Green Bee-Eater & Merops orientalis & Insects \\
\hline 40 & Motacillidae & White Wagtail & Motacilla alba & insects \\
\hline 41 & Motacillidae & Pipit sp. & Anthus & Insects \\
\hline 42 & Muscicapidae & Common Stone Chat & Saxicola torquatus & insects \\
\hline 43 & Muscicapidae & Indian Robin & Saxicoloides fulicata & Insects \\
\hline 44 & Nectariniidae & Purple Sunbird & Cinnyris asiaticus & Mainly nectar, also insects \\
\hline 45 & Passeridae & Chestnut-shouldered Petronia & Passer domesticus & Grains, Seeds \\
\hline 46 & Phalacrocoracidae & Little Cormorant & Phalacrocorax niger & Fish \\
\hline 47 & Phasianidae & Grey Francolin & Francolinus pondicerianus & $\begin{array}{l}\text { Mainly vegetable matter, } \\
\text { some amount of insects }\end{array}$ \\
\hline 48 & Phasianidae & Indian Peafowl & Pavo cristatus & $\begin{array}{l}\text { Mainly plant matter, but } \\
\text { also insects }\end{array}$ \\
\hline 49 & Ploceidae & Black-breasted Weaver & Ploceus benghalensis & Seeds, grains \\
\hline 50 & Psittacidae & Rose-ringed Parakeet & Psittacula krameri & Plant matter \\
\hline 51 & Pycnonotidae & Red-vented Bulbul & Pycnonotus cafer & Plant matter and insects \\
\hline 52 & Strigidae & Spotted Owlet & Athene brama & $\begin{array}{l}\text { Rodents, reptiles, birds, } \\
\text { insects }\end{array}$ \\
\hline 53 & Sturnidae & Bank Myna & Acridotheres ginginianus & Insect, grains, Fruits \\
\hline 54 & Sturnidae & Common Myna & Acridotheres tristis & Mainly Insects \\
\hline
\end{tabular}




\begin{tabular}{|c|l|l|l|l|}
55 & Sturnidae & Rosy Starling & Pastor roseus & Insects, seeds etc. \\
\hline 56 & Threskiornithidae & Black Ibis & Pseudibis papillosa & Insects \\
\hline 57 & Threskiornithidae & Red-naped Ibis & $\begin{array}{l}\text { Threskiornis } \\
\text { melanocephalus }\end{array}$ & $\begin{array}{l}\text { Various invertebrates, } \\
\text { including insects }\end{array}$ \\
\hline 58 & Threskiornithidae & Black-headed Ibis & $\begin{array}{l}\text { Threskiornis } \\
\text { melanocephalus }\end{array}$ & $\begin{array}{l}\text { insect not main, but a part } \\
\text { of the diet }\end{array}$ \\
\hline 59 & Zosteropidae & OrienStel White- eye & Zosterops palpebrosus & Mainly Insects \\
\hline
\end{tabular}

*Food items were determined through literature survey

The occurrence of 59 species of birds in approximately 5 sq.km area of the airport premises can be considered relatively high bird species richness in a non-winter season (which is known for good influx of migratory birds in various parts of the state). This is because SVP International airport is situated amidst the buzzing suburbs of Ahmedabad city like Kubernagar, Meghaninagar, Sardarnagar, Camp Hanuman etc. These areas have numerous roads with busy traffic, residential societies, and commercial buildings. Just adjacent to some portions of the boundary wall of the airport falling in areas like Kubernagar and Krishnanagar, densely packed buildings of some residential societies existed overlooking the airport premises. So, it was a riddle why species richness as high as 59 bird species occurred on the airport premises which included even rural and/or grassland birds like Grey Francolin, Ashy-crowned Sparrow-Lark, Rufous-tailed Lark, Blue-tailed Bee-eater, raptors etc. Therefore, investigations were carried out to understand or infer the reason for such high bird diversity on SVP International airport despite its location in the city's suburban area. The investigations regarding the obligatory or facultative food of each of the species through review of the relevant and authentic literature [12].

The investigation revealed that 49 species (i.e., 83\%) of the total bird species recorded on the airport premises were those known to be either obligatorily or facultatively insect-eaters. The field monitoring on insect diversity on airport premises (along with the bird surveys along two bird transects and at random bird observation points) showed that a total of 80 species of insects occurred on the SVP International airport that belonged to 42 families and 11 orders (Table 2).

The highest number of insect species belonged to the Order Lepidoptera, i.e. $27 \%$ of total species composition(including 22 species of moths and butterflies) followed by Orthoptera i.e $15 \%$ of total species composition (including 12 species of grasshoppers and locusts). Relatively lesser number of species of the orders Embioptera, Isoptera (including termites) and Neuroptera (including Antlion) were encountered (Fig 4).

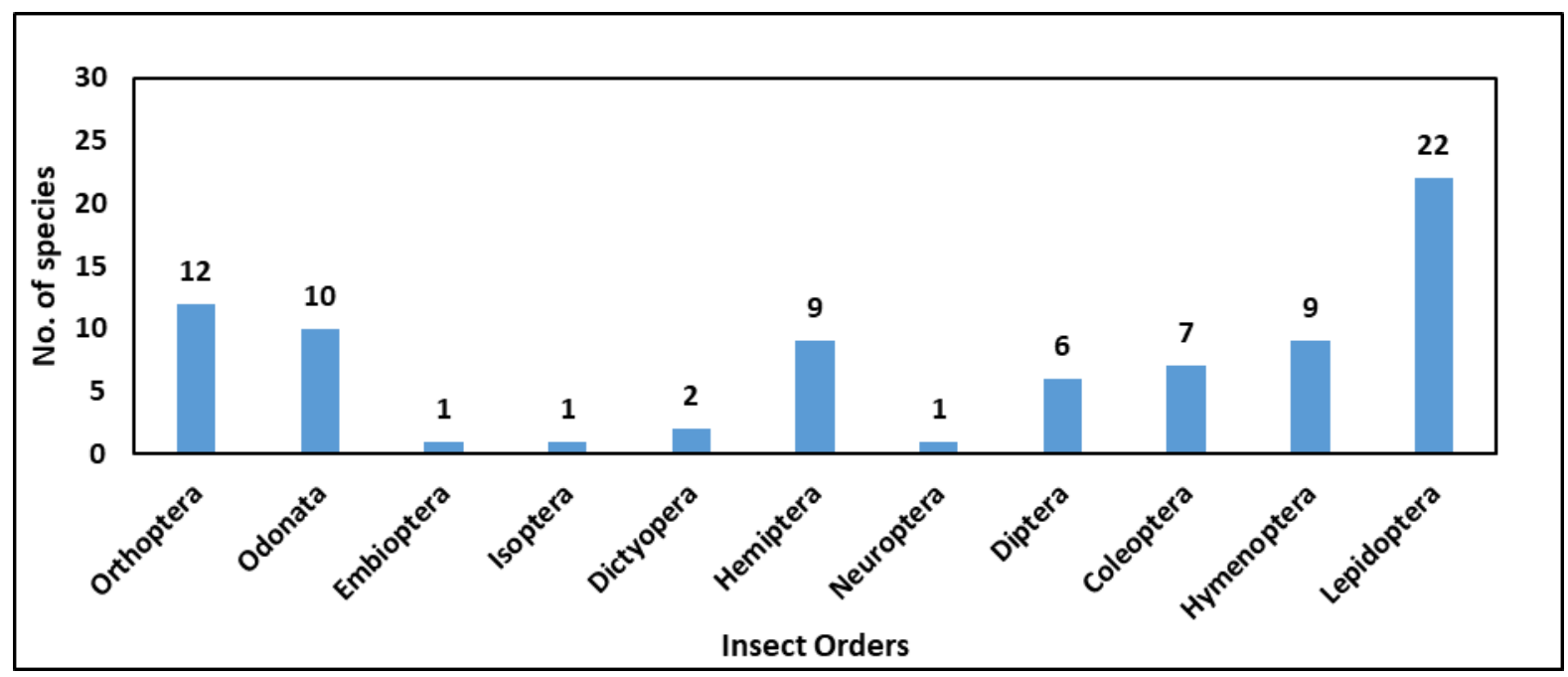

Fig 4. Orderwise summary of the number of insect species at SVPI Airport (2015-2017)

Table 2: Checklist of insects encountered at SVP International airport (2015-2017)

\begin{tabular}{|c|c|c|}
\hline Order & Family & Insect Species \\
\hline
\end{tabular}




\begin{tabular}{|c|c|c|}
\hline \multirow[t]{3}{*}{ Orthoptera } & Acrididae & $\begin{array}{l}\text { Amblytropidia mysteca (Saussure, 1861),Schistocerca } \text { gregaria } \text { (Forsskal, } \\
\text { 1775),Hieroglyphus banian(Fabricius, 1798),Acrida conica (Fabricius, } \\
\text { 1781),Acrida ungarica (Herbst, 1786), Acrida species, Chorthippus } \\
\text { biguttulus (Linnaeus, 1758),Omocestus } \\
\text { Choroedocus sp. }\end{array}$ \\
\hline & Pyrgomorphidae & $\begin{array}{l}\text { Poekiloceru spictus(Fabricius, 1775), Atractomorpha crenulata(Fabricius, } \\
\text { 1793) }\end{array}$ \\
\hline & Tetrigidae & Paratettixsp. \\
\hline \multirow[t]{2}{*}{ Odonata } & Coenagrionidae & $\begin{array}{l}\text { Ischnura aurora (Brauer, 1865),Ceriagrion coromandelianum (Fabricius, } \\
\text { 1798) }\end{array}$ \\
\hline & Libellulidae & $\begin{array}{l}\text { Trithemis pallidinervis(Kirby, 1889), Pantala flavescens } \text { (Fabricius, } \\
\text { 1798),Brachythemis contaminata (Fabricius, 1793), Crocothemis } \\
\text { servilia(Drury, 1770) ,Bradinopyga geminate (Rambur, 1842),Orthetrum } \\
\text { sabina(Drury, 1770),Trithemis aurora (Burmeister, 1839),Diplacodes } \\
\text { trivialis (Rambur, 1842) }\end{array}$ \\
\hline Embioptera & Embiidae & Embia sp. \\
\hline Isoptera & Rhinotermitidae & Reticulitermes flavipes(Kollar, 1837) \\
\hline \multirow[t]{2}{*}{ Dictyopera } & Blattidae & Periplaneta americana (Linnaeus, 1758) \\
\hline & Mantidae & Mantis religiosa(Linnaeus, 1758) \\
\hline \multirow[t]{8}{*}{ Hemiptera } & Aphididae & Aphis nerii (Boyer de Fonscolombe, 1841) \\
\hline & Cercopidae & Philaenus spumarius (Linnaeus, 1758) \\
\hline & Cicadidae & Platypleura octoguttata (Fabricius, 1798) \\
\hline & Coreidae & Cletus bipunctatus (Herrich-Schäffer, 1840) \\
\hline & Curculionidae & Conotrachelus posticatus (Boheman, 1837) \\
\hline & Lygaeidae & Lygaeus kalmii (Stal 1874), Lygaeus hospes (Fabricius, 1794) \\
\hline & Pentatomidae & Eysarcoris sp. \\
\hline & Pyrrhocoridae & Dysdercus cingulatus (Fabricius, 1775). \\
\hline Neuroptera & Myrmeleontidae & Myrmeleon sp. \\
\hline \multirow[t]{6}{*}{ Diptera } & Asilidae & Promachus sp. \\
\hline & Bombyliidae & Bombus sp. \\
\hline & Calliphoridae & Calliphora vomitoria (Linnaeus, 1758) \\
\hline & Muscidae & Musca domestica (Linnaeus 1758) \\
\hline & Sarcophagidae & Sarcophaga sp. \\
\hline & Tephritidae & Bactrocera sp. \\
\hline \multirow[t]{6}{*}{ Coleoptera } & Buprestidae & Buprestis aurulenta (Linnaeus, 1767) \\
\hline & Carabidae & Brachinus sp. \\
\hline & Chrysomelidae & Charidotella sp., Crioceris sp. \\
\hline & Curculionidae & Artipus sp. \\
\hline & Geotrupidae & Anoplotrupes sp. \\
\hline & Meloidae & Mylabris pustulata (Thunberg, 1821) \\
\hline \multirow[t]{3}{*}{ Hymenoptera } & Apidae & $\begin{array}{l}\text { Apis cerana indica (Fabricius, 1798), Amegilla cingulate (Fabricius, 1775), } \\
\text { Xylocopa auripennis (Lepeletier, 1841) }\end{array}$ \\
\hline & Braconidae & Opius sp. \\
\hline & Formicidae & $\begin{array}{l}\text { Camponotus compressus (Fabricius, 1787), Monomorium minimum (Buckley, } \\
\text { 1867) }\end{array}$ \\
\hline
\end{tabular}




\begin{tabular}{|c|c|c|}
\hline & Vespidae & $\begin{array}{l}\text { Polistes wattii (Cameron, 1900), Icaria variegata (Smith, 1852), Delta } \\
\text { esuriens (Fabricus 1787) }\end{array}$ \\
\hline \multirow[t]{8}{*}{ Lepidoptera } & Nymphalidae & $\begin{array}{l}\text { Danaus chrysippus (Linnaeus, 1758), Acraea terpsicore(Linnaeus, 1758), } \\
\text { Hypolimnas misippus(Linnaeus, 1764), Junonia almana(Linnaeus, 1758), } \\
\text { Junonia orithya(Linnaeus, 1758) }\end{array}$ \\
\hline & Papilionidae & $\begin{array}{l}\text { Papilio demoleus(Linnaeus, 1758), Graphium agamemnon (Linnaeus, 1758), } \\
\text { Pachliopta aristolochiae(Fabricius, 1775) }\end{array}$ \\
\hline & Pieridae & $\begin{array}{l}\text { Catopsilia pomona } \text { (Fabricius, 1775), Eurema hecabe, Colotis } \\
\text { amata(Fabricius, 1775), Ixias marianne(Cramer, 1779) }\end{array}$ \\
\hline & Lycaenidae & $\begin{array}{l}\text { Catochrysops strabo(Fabricius 1793), Chilades trochylus(Freyer } 1845) \text {, } \\
\text { Tarucus plinius(Fabricius, 1793), Zizina otis(Fabricius, 1787), Tarucus } \\
\text { nara(Kollar, 1848), Zizula hylax(Fabricius, 1775) }\end{array}$ \\
\hline & Hesperiidae & Borbo cinnara (Wallace, 1866) \\
\hline & Erebidae & Pyrrharctia Isabella (JE Smith, 1797) \\
\hline & Geometridae & Hyperythra lutea(Stoll, 1781) \\
\hline & Pterophoridae & Stenoptilia sp. \\
\hline
\end{tabular}

The population of insects(irrespective of the species) was high too, especially in monsoon season. During monsoon season, average insect population (in total 8 quadrats of $1 \mathrm{mx} 1 \mathrm{~m}$ laid at equal distance for along the transects walked for birds) for the years between 2015 and 2017 was found to be 928 individuals. Though during premonsoon, the average insect population was relatively low $(\mathrm{n}=230)$ in total 8 equi-distantly laid $1 \mathrm{mx} 1 \mathrm{~m}$ quadrats along the bird transects.

High species diversity and high insect population during monsoon can be easily linked with good habitat conditions for entomofauna on the airport premises during pre-monsoon to monsoon each year. Good habitat conditions were found to get created due to good grass and herb cover especially in monsoon in many portions of the airport premises away from the runway. With few isolated showers even in pre-monsoon season (June), grass and herb growth was found to get initiated and that reached the peak during monsoon leading to high insect population in monsoon. Grasses and some herbs grew like weeds on the airport premises despite repeated efforts of the SVPI airport authorities to arrange for herb and grass harvesting mechanically. Weeds are known to be a primary food resource for many phytophagous insects [13]. Grass and herbaceous cover on the airport premises might also have provided cover to many insects so that they would not get directly exposed to insectivorous birds. The study has led to an inference that one of the reasons of good bird diversity on the airport premises may be due to a higher proportion of insectivorous bird species, which in turn might be due to high diversity and a good population of insects owing to grass and herb cover on the airport premises. Termite nests were found to exist beneath the weed cover and termites are known to be a good proteinaceous food for many birds including Black Kite (Milvus migrans). It is usually believed that Black Kites only feed on offals, but that is not true. Winged termites form a favorite food for Black Kites especially in monsoon when several kites are seen busy capturing winged termites in mid-air. Termites usually occur in large numbers and therefore they can easily draw the attention of foraging birds like Black Kites. In turn, they may get attracted towards the airport premises leading to increased chances BASH. The weed cover also provided good habitat for small rodents (e.g., rats and mice) and reptiles (e.g., lizards) and they also constitute food for Black Kites. Thus, it is likely that at SVP International airport, Black Kites have been getting offal from the careless solid waste dumping in the vicinity of the airport and in addition, they also might be getting termites, other insects and small rodents and reptiles on the airport premises. Therefore, Black Kite was found to be the most dominant/abundant bird on the SVPI airport premises. Table 3 indicates that Black Kite has not only been among the top 5 most abundant bird species(i.e., Black Kite, Cattle Egret, Blue Rock Pigeon, House Crow and Common Myna), but it has been the only species that had occurred in relatively good numbers irrespective of season (pre-monsoon and monsoon) at Ahmedabad airport. During the bird monitoring on airport premises, Black Kite was often found soaring/gliding over the run-way and it was also found to sit or devour prey on run-way. It was found that the kite had got used to the movement of aircrafts landing and taking-off at the airport making it one of the most hazardous bird at SVP International airport. 
Table 3: Seasonal population monitoring of bird species encountered along two transects in SVP International airport operation area Ahmedabad

\begin{tabular}{|c|c|c|c|c|c|}
\hline \multirow{3}{*}{ Sr. No. } & \multirow{3}{*}{ Common name } & \multicolumn{4}{|c|}{ Number of Birds } \\
\hline & & \multicolumn{2}{|c|}{ Monsoon season } & \multicolumn{2}{|c|}{ Premonsoon season } \\
\hline & & Average & Highest & Average & Highest \\
\hline 1 & Rosy Starling & 2513 & 5000 & 0 & 0 \\
\hline 2 & Cattle Egret & 105 & 203 & 15 & 19 \\
\hline 3 & Blue Rock Pigeon & 87 & 163 & 22 & 30 \\
\hline 4 & Black Kite & 75 & 102 & 43 & 57 \\
\hline 5 & House Crow & 29 & 52 & 10 & 16 \\
\hline 6 & Common Myna & 18 & 30 & 18 & 22 \\
\hline 7 & Black-headed Ibis & 16 & 24 & 4 & 8 \\
\hline 8 & Red-wattled Lapwing & 14 & 15 & 17 & 29 \\
\hline 9 & Green Bee-eater & 12 & 17 & 6 & 8 \\
\hline 10 & Black Drongo & 11 & 17 & 10 & 17 \\
\hline 11 & Rose-ringed Parakeet & 10 & 17 & 7 & 11 \\
\hline 12 & Bank Myna & 10 & 13 & 9 & 12 \\
\hline 13 & House Sparrow & 9 & 14 & 1 & 1 \\
\hline 14 & Indian Silverbill & 7 & 9 & 0 & 0 \\
\hline 15 & Red-vented Bulbul & 6 & 7 & 5 & 8 \\
\hline 16 & ZittingCisticola & 5 & 9 & 7 & 9 \\
\hline 17 & Eurasian Collared Dove & 5 & 7 & 2 & 3 \\
\hline 18 & Laughing Dove & 5 & 7 & 3 & 5 \\
\hline 19 & Large Grey Babbler & 5 & 5 & 1 & 2 \\
\hline 20 & Blue-tailed Bee-eater & 5 & 1 & 2 & 3 \\
\hline 21 & Grey Francolin & 5 & 7 & 6 & 7 \\
\hline 22 & Indian Peafowl & 5 & 5 & 0 & 0 \\
\hline 23 & Ashy Prinia & 4 & 4 & 2 & 2 \\
\hline 24 & Common Tailorbird & 4 & 7 & 2 & 3 \\
\hline 25 & White-throated Kingfisher & 3 & 4 & 1 & 2 \\
\hline 26 & Dusky Crag Martin & 3 & 6 & 1 & 1 \\
\hline 27 & Red-naped Ibis & 3 & 6 & 2 & 3 \\
\hline 28 & Oriental White-eye & 3 & 6 & 0 & 0 \\
\hline 29 & Rufous-fronted Prinia & 3 & 5 & 1 & 2 \\
\hline 30 & Indian Roller & 3 & 4 & 1 & 1 \\
\hline 31 & Indian Robin & 3 & 4 & 4 & 5 \\
\hline 32 & Ashy-crowned Finch- Lark & 2 & 4 & 2 & 4 \\
\hline 33 & Grey-breasted Prinia & 2 & 4 & 1 & 2 \\
\hline
\end{tabular}




\begin{tabular}{|c|c|c|c|c|c|}
\hline 34 & Asian Koel & 2 & 2 & 1 & 2 \\
\hline 35 & Greater Coucal & 2 & 2 & 1 & 1 \\
\hline 36 & Wire-tailed Swallow & 2 & 4 & 0 & 0 \\
\hline 37 & Common Babbler & 2 & 4 & 0 & 0 \\
\hline 38 & Purple Sunbird & 2 & 3 & 2 & 3 \\
\hline 39 & Spotted Owlet & 2 & 3 & 0 & 0 \\
\hline 40 & Shikra & 1 & 1 & 2 & 2 \\
\hline 41 & Indian Pond Heron & 1 & 1 & 1 & 1 \\
\hline 42 & Long-tailed Shrike & 1 & 2 & 0 & 0 \\
\hline 43 & Black-breasted Weaver & 1 & 2 & 0 & 0 \\
\hline 44 & Black-shouldered Kite & 1 & 1 & 0 & 0 \\
\hline 45 & Yellow-wattled Lapwing & 1 & 1 & 3 & 4 \\
\hline 46 & Rufous Treepie & 1 & 1 & 1 & 1 \\
\hline 47 & Little Swift & 0 & 0 & 1 & 2 \\
\hline 48 & Little Cormorant & 0 & 0 & 1 & 1 \\
\hline
\end{tabular}

*Note: The sequence of the species name arranged in the descending order of number of individuals encountered

Table 3 also indicates that Rosy Starling(Pastor roseus), an early immigrant to Gujarat each year has occurred in massive numbers (upto 5000 individuals), but only during monsoon. Rosy Starling feeds on insects like locusts and grasshoppers beside plant-based food. Rosy Starling can create a problem for an aircraft due to their habit of flying in huge flocks. Red-wattled Lapwing(Vanellus indicus) was the only insectivorous bird that is known to actively forage during the night also and thus it has the potential to cause trouble to the air-crafts landing or taking-off at nights. If individuals of this species are allowed to nest on the airport operational area, they can be more troublesome for the aircraft.

\section{CONCLUSION AND RECOMMENDATIONS}

SVP International Airport supports a large number of bird species in pre-monsoon to monsoon season as the airport attracts large number insectivorous bird species owing to the fast-growing weed cover that exists on the airport premises that supports high insect diversity and population. During a study on birds at Ahmedabad airport (SVP International Airport) and its environs had indicated that Black Kite occurs on the airport premises in good numbers irrespective of the season (pre-monsoon or monsoon) [7].

Usually, every animal mainly were drawn to an area due to the availability of preferred food and habitat. for minimizing the potential of bird-aircraft collision hazards at SVP International airport, various measures can be planned and implemented such as a) regular harvesting of grass and herb cover (i.e., weed cover) for discouraging insect occurrence, b) controlling population of rodents, c) proper control of termites on the airport premises as they constitute one of the food items of Black Kites and other birds can be done to keep away these birds, d)explore the possibility of grass harvesting/cutting at night or during crepuscular (dawn/duck) hours as kites and other birds preferring insects and other small animals as food opportunistically flocks at the harvesting spots during day-time, e) maintaining the cover of only single grass species as a part of habitat management and f) using artificial grass/turf cover instead of currently existing natural/wild grass cover.

SVP International Airport has taken a proper initiative by roping in GEER Foundation for conducting an investigation on birds, insects and other small fauna, a step towards reducing BASH. All other airports may follow this step considering the seriousness of the issue.

\section{ACKNOWLEDGMENTS}

Authors are thankful to the authorities of SVPI Airport and AEMC for assigning the research work and give all the necessary logistic support during every field investigation. Authors are also thankful to Irshad Theba, Technical Assistant (Bird Breeder), GEER Foundation and Darpak Joshi, Researcher GEER Foundation for field Survey pertaining to birds. 


\section{REFERENCES}

[1]. L. A. Dale, "Personal and corporate liability in the aftermath of bird strikes: A costly consideration". Human-Wildlife Conflicts, 3 (2), $216-$ 225,2009

[2]. R. A. Dolbeer, and S. E. Wright, "Safety management systems: how useful will the FAA national wildlife strike database be?" Human-wildlife conflicts 3 pp. 167-178, 2009.

[3]. Maragakis, "Bird population trends and their impact on aviation safety 1999-2008". Safety report European aviation safety agency, safety analysis and research department, pp. 1-20, 2009.

[4]. U. C. Bernice., and J. K. Floyd, "An Avian/Airport Study for Standiford Airport, Louisville, Kentucky Results And Management Implications" In: 5 - Fifth Eastern Wildlife Damage Control Conference. pp 1-12, 1991.

[5]. R. E. Harris, \& R. A. Davis, "Evaluation of the efficacy of products and techniques for Airport Bird Control". LGL Report TA2193. LGL Limited, Environmental Research Associates. 1998.

[6]. G.R., Davidson, Jr., T.V. Degeare, Jr., T.J. Sorg, and R.M. Clark. "Land disposal near airports reporting bird/aircraft hazards" U.S. Environmental Protection Agency. Open-file Report (TSR 1.1.004/0) 1971.

[7]. B. J. Pathak, K. Tatu, V. Vyas, and K. Mokaria, "A Study on Birds at Ahmedabad Air-port (SVPI Airport) \& its Environs to Minimize Bird-strike Hazards to Aircrafts [with Emphasis on Problems from Black Kite (Milvus migrans)]", Report no. 68/2013, Gujarat Ecological Education and Research (GEER) Foundation, Indroda Nature Park, Gandhinagar, Gujarat Technical Report, pp. 1-86, 2012.

[8]. D. Borer, N. Johnson, and C. Triplehorn "Borror and DeLong's An introduction to the study of insects", Saunders collage publication, Ohio State University, OH, USA No. Ed. 6, pp 1-875, 1989.

[9]. P. S., Corbet, "Dragonflies: Behaviour and Ecology of Odonata" Comstock Publishing Associates, Ithaca, New York, pp. 1-830, 1999.

[10]. A.D. Imms, O.W. Richards, R. G. Davies, (Eds.) Imms' "General Textbook of Entomology Volume 2: Classification and Biology, Springer, Dordrecht, ISBN 978-94-011-6516-7, Volume 2 pp. 1-1279, 1977.

[11]. K. A. Subramanian, Dragonflies and Damselflies of Peninsular India-A Field Guide. E-Book of Project Lifescape. Centre for Ecological Sciences, Indian Institute of Science and Indian Academy of Sciences, Bangalore, India. pp. 1-118. 2005.

[12]. S. Ali, and S. D. Ripley, "Handbook of the Birds of India and Pakistan". (Vol.1-10). Oxford University Press, New Delhi, 2002.

[13]. J. L. Capinera, "Relationships between insect pests and weeds: an evolutionary perspective”. Weed Science 53, pp. 892-901, 2005.

\section{Author's Profile}

Kalavanti Mokaria is an entomologist at GEER Foundation, Gandhinagar, Gujarat, India. She has completed PostGraduation in Zoology with specialization in Entomology. She has done research in the field of biodiversity with emphasis on Entomology in various ecosystems including forest, grasslands and freshwater wetlands and urban area. She also has work experience in laboratory research and had been studying chemical ecology of insects. She has been contributing to environmental education and awareness programme through radio talks and Nature Education camps. She is an Executive Council member of Indian Dragonfly Society (IDS) and also involved in insect surveys within India.

Dr. Ketan Tatu is the Senior Scientist, Gujarat Ecological Education and Research (GEER) Foundation, Gandhinagar. He has earned a Ph.D. in Environment Science from Gujarat University, India and a Ph.D. in Forest Resources Science, West Virginia University, USA. He is a Member of State Wetland Authority of Gujarat State and a Member of SACON Society. Dr. Tatu is the Founding Editor of "Jalaplavit" (ISSN 2321-1881)- an e-Periodical to enhance the knowledge about wetland biodiversity among people and sensitize them for its conservation. He is also a writer of an on-going column "Lap of Nature" in a newspaper "Daily News \& Analysis" (DNA) that is aimed at inculcating interest in Nature and appreciation for it among laymen. He has authored/co-authored many scientific papers, technical reports and book chapters in the field of wetlands and birds. Dr. Ketan Tatu is a Life Member of Bombay Natural History Society (BNHS) and Bird Conservation Society, Gujarat (BCSG).

Mr. R. D. Kamboj is an Indian Forest Services Officer of Gujarat cadre (1986 batch) of the rank of Additional Principal Chief Conservator of Forests. He holds post graduate degree in Agriculture from Haryana Agriculture University, Hisar \& in Forestry from Indira Gandhi National Forest Academy, Dehradun. He has served in various positions in Gujarat Forest Department for last 32 years. He has published more than 60 research papers and has written Working Plans/ Management Plans for various forest divisions including Gir National Park \& Sanctuary. Presently he is serving as Director, Gujarat Ecological Education and Research (GEER) Foundation, Gandhinagar.
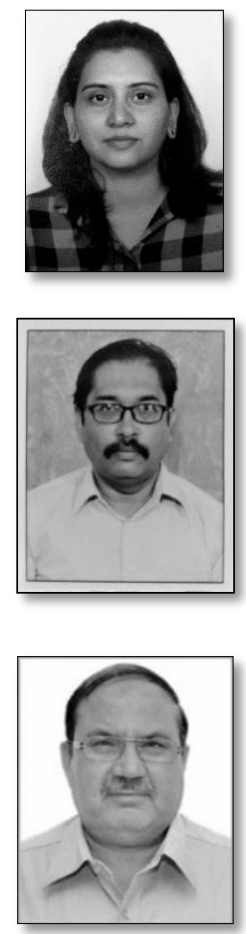